\title{
La tecnología como proceso de producción del espacio
}

\section{Technology as a process of production of space}

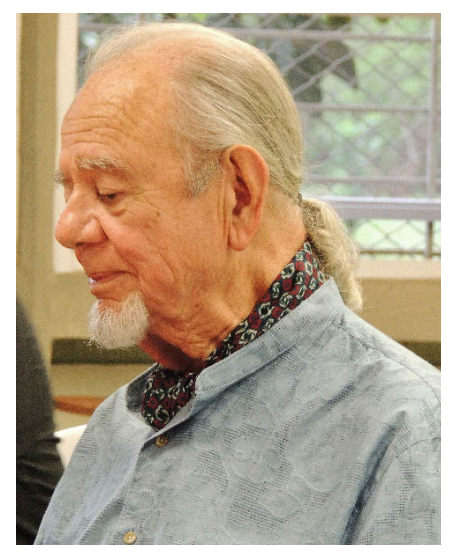

\section{Otto Vallderuten Daraviña}

Universidad del Valle, Cali, Colombia.

Correo electrónico: ottovallderuten@gmail.com

Fuente: 70 años de la Escuela de Arquitectura de la Facultad de Artes Integradas (2017), ponencia Tecnológica de Arquitectura a cargo del arquitecto Otto Vallderuten Daraviña

\section{Presentación}

Arquitecto Universidad del Valle, 1959. Desarrolló varios proyectos con el grupo interdisciplinario conformado para el diseño de la ciudad Est Halle (Alemania) entre 1964 y 1968. Ha sido profesor del Departamento de diseño de la Escuela de Arquitectura de la Universidad Nacional de Colombia. Profesor de la Facultad de Arquitectura de la Universidad de los Andes (Colombia). Profesor titular de la Universidad del Valle, adscrito al Departamento de Tecnología de la Escuela de Arquitectura (Facultad de Artes Integradas) desde 1988. Asesor del Plan de desarrollo de Cali de 1990, siendo autor de los "Cuadernillos del Plan de Desarrollo". Realizó el Estudio del Plan Vial de Cali (1969).

La Tecnología se ha convertido en una de las preocupaciones de la vida contemporánea y no es para menos.

Voy a intentar, con ánimo pedagógico, acercar el conflicto que surge cuando no existe claridad entre un oficio en particular y la tecnología que demanda.

Tal el caso de la arquitectura donde la tecnología, para algunos, es un recurso externo que se toma o se deja según el criterio del oficiante de un objeto sencillo o de un complejo urbano.

Para complicar aún más este conflicto del conocimiento contemporáneo, algunos afirman que la tecnología es una especie de ciencia pequeña y que aparece a partir de postulados verdaderos o se deriva a partir de ellos.

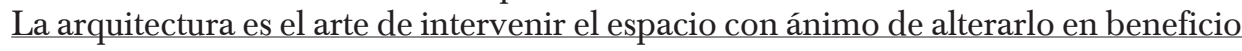
del hombre, en particular, o de la sociedad en general. 
Desde el hombre primitivo hasta nuestros días este proceso productivo, social, de intervención del espacio, constituye un continuo sin final, un continuo cada vez más complejo, como complejas se hacen las acciones del hombre.

Digamos, en términos contemporáneos, que la arquitectura se enraíza con la totalidad de los procesos de la sociedad, a manera de síntesis de la complejidad de la misma.

La tecnología, muy a diferencia de las diversas definiciones de los legos, no es otra cosa que el "proceso de producción".

No es otra cosa que el conjunto de eventos interrelacionados que conduce a la producción de un bien final.

Bien final éste que puede ser un objeto o un servicio.

Digamos, entonces, que la arquitectura implica una tecnología que conduce a la producción del bien final arquitectónico.

Habrá por lo tanto una tecnología propia de la producción del espacio, que paralelamente al desarrollo histórico ha cambiado continuamente para lograr el producto arquitectónico contemporáneo cada vez otro, cada vez más complejo, cada vez más audaz, en la medida en que los eventos se dan en procesos más complejos y audaces.

En síntesis, que la tecnología no es otra cosa que "el proceso productivo" mismo, tal como lo definieran los clásicos de la economía moderna y contemporánea.

Un proceso productivo propio de cada bien o servicio que la sociedad demanda y produce.

No existe una tecnología única, pues cada bien o producto exige para su materialización un proceso específico.

Ese proceso específico en la producción del hecho arquitectónico, constituye la tecnología arquitectónica. Proceso éste que ha venido mutando y cambiando en la medida en que los requerimientos de la vida contemporánea lo exigen.

El hecho arquitectónico supone la utilización de otras tecnologías complementarias a la producción de la diversidad de insumos y servicios necesarios en el proceso de materialización del producto arquitectónico.

Esos procesos que acuden o intervienen en el "hecho arquitectónico", urbano o territorial, son buena parte de los procesos sociales que intervienen en los sectores de la economía global, vale decir, el sector primario, secundario y terciario.

Casi que podríamos decir que en la tecnología arquitectónica se refleja la complejidad de la producción social de bienes y servicios que a su vez implicaron el desarrollo de otras tecnologías.

La Tecnología es, a diferencia de la ciencia, una categoría cambiante que se acomoda a las necesidades del momento y por tanto es objeto continuo de reformulación y cambio.

La Tecnología arquitectónica posee esa característica de "cambiante" en la medida en que los eventos propios de su proceso son susceptibles de modificaciones permanente.

Lo más importante que deseamos consignar en el hecho que de la tecnología es susceptible de ser diseñada y ésta constituye una de las tareas más significativas en la actividad creativa del arquitecto contemporáneo.

Formulemos la primera pregunta para provocar la reacción del auditorio. ¿Será 
posible satisfacer una demanda arquitectónica sin saber correctamente cuál es el proceso productivo adecuado, sin saber la tecnología conveniente al objeto a diseñar?

$\mathrm{O}$ formulada de manera diferente.

¿Es posible concebir un espacio arquitectónico sin comprometerse con su proceso de materialización?

Esta simbiosis entre forma y proceso productivo enriquece los ritmos compositivos en la medida en que se corresponden, con el tiempo del ajuste entre las partes y el todo constructivo. Las partes de la composición se ajustan con los tiempos y ritmos de ejecución.

Lamentablemente, tenemos que decir que la mayoría de las arquitecturas en nuestro país se formulan para que después del proceso licitatorio o del concurso, otras personas, ajenas al proceso compositivo, definan, sin necesidad previa de describirlo, como será materializada esa idea.

Después del concurso, después de la licitación, un oferente, un constructor, define el proceso productivo que ha de materializar esa idea a su amaño y según sus intereses económicos, que no necesariamente coinciden con el mejoramiento estético de la obra.

Esta dicotomía entre proceso de concepción de la idea arquitectónica y su materialización constituye una de las lamentables razones para la mediocridad de buena parte de la producción arquitectónica entre nosotros y porque no decirlo a escala nacional.

En esta charla me propongo llevar a ustedes la idea de que el proceso compositivo debe llevar paralelo (y de mano del autor), el proceso tecnológico, vale decir, pensar y hacer en una síntesis totalizadora. La tecnología interrelaciona para la producción del bien o servicio final, cinco variables en el proceso, a saber:

A) La historia, la cultura, la geografía y la antropología, que podemos llamar "El hombre cultural”.

B) Los recursos de capital. El ahorro, la acumulación que podríamos llamar `El hombre acumulador'.

C) La organización empresarial, que podríamos llamar "El capital humano".

D) Las herramientas para incrementar la productividad y reducir la mano de obra $\mathrm{y}$ afinar el producto final.

E) Los materiales que sustentan la materialidad del producto y caracterizan la unidad de concepción.

- Cada grupo, humano según la geografía de su ubicación, la historia de su vida productiva, deja rasgos antropológicos inconfundibles en los productos para el uso y para el intercambio.

- Frente a la definición de cada proceso productivo hemos de preguntarnos. ¿Qué impronta histórica geográfica llevarán los productos de un proceso particular?

- En cada país, en cada sociedad, hemos de preguntarnos, obligatoriamente, por las formas de acumulación de capital que participan o hacen posible un proceso específico de producción y cuales las más convenientes a un proceso determinado.

La caracterización del tipo y forma de capital conveniente para un proceso juegan un papel determinado en el resultado o producto final.

- La organización empresarial responderá por la mayor o menor participación de personal especializado y esa caracterización del capital humano indispensable a un 
proceso específico sustenta una tecnología en particular y responde por el resultado del producto final.

- Las herramientas, su mayor o menor complejidad caracterizarán una tecnología en términos de rendimiento, eficiencia, precisión y velocidad de producción y calidad del producto final.

- Los materiales necesarios a un proceso, la diversidad de los mismos y sus características relevantes definen los límites y la forma de los productos finales esperados, así como las calidades estéticas de los mismos.

La interrelación de estas cinco variables caracteriza en toda su amplitud, un proceso de producción. La mayor o menor influencia o participación de cada uno de ellos, dará origen a procesos diversos y por tanto a productos diversos.

La alteración de la participación de una variable conducirá necesariamente a productos nuevos y diferentes.

La innovación, preocupación fundamental de la vida y la producción contemporánea, no es otra cosa, que es resultado de la alteración de la participación de todas o alguna de estas cinco variables en el proceso.

La alteración del proceso conduce a la oferta de nuevos productos. Así como la necesidad de nuevos productos conduce a una modificación del proceso.

En la arquitectura, la tecnología apropiada viene a ser responsable de la "diversidad de la producción”. Condición indispensable en los mercados contemporáneos, modernos, donde el diseñador es responsable de ofrecer "diversidad en la producción".

Las Tecnologías reciben nombres que hacen relación a la predominancia de alguna de las variables del proceso.

- El ámbito geográfico de un desarrollo conduce a la denominación por países de la tecnología. Así encontramos tecnología alemana, japonesa y americana según el país de sus productos finales.

- Según la necesidad de capital tendremos tecnologías de alta o baja composición de capital.

- Según las necesidades de mano de obra tenemos tecnologías de baja o alta composición o de alta o baja especialización.

Igualmente tendremos tecnologías de baja o alta composición de capital humano.

- Las herramientas darán igualmente nombre a las tecnologías según la baja o alta exigencia de herramientas hasta la robotización.

- Los materiales predominantes darán igualmente nombre a tecnologías metálicas, de acero, de concreto, igualmente de baja o alta participación de un material específico.

Los modelos de repetición lineal indefinida de soluciones arquitectónicas, sobre todo en la oferta de proyectos sociales, no es más que deficiencia del proceso productivo, falta de la tecnología apropiada que permita en un mismo proceso una diversificación de los productos finales que se corresponda con la diversidad de condiciones del mercado o usuarios finales, en términos de ingresos, de ahorro, de composición familiar, de hábitos o costumbres.

La aparición de nuevas formas de organización de las empresas constructoras, hace prever junto a una mayor participación de personal calificado en la formulación del proceso frente al uso de materiales adecuados y herramientas de alto rendimiento, 
podrá garantizar una velocidad de producción compatibles con los costos del capital a invertir por unidad de tiempo.

Igualmente, la definición del entramado arquitectónico compositivo ligada a la estructuración de los esfuerzos que garantizan la estabilidad y la unidad del todo, superando la falsa dicotomía entre arquitectos y estructura tan dañina a una arquitectura seria.

La definición paralela del proceso compositivo y de la tecnología, garantizan la aplicación racional de los recursos en la materialización de la idea y la unidad del hecho arquitectónico.

La ciudad es la forma más compleja de la producción humana, es la expresión más dramática de la diversidad de ofertas que la vida social brinda al hombre y su comunidad.

La ciudad es el resultado de un complejo fenómeno de gestión racional, organizada, a la par de la mayor espontaneidad productiva que haya experimentado el hombre.

Esas Tecnologías que racionalizan la complejidad de estos procesos de urbanización, se denominan. "Tecnologías urbanas" y se encargan de garantizar la producción de todos los recursos necesarios a la vida urbana.

El tamaño de las ciudades y su importancia se definen por el nivel de desarrollo de esos procesos que se encargan de brindar servicios a su población.

El grado de desarrollo de esas infraestructuras caracteriza el grado de importancia de las ciudades y el nivel de su desarrollo.

Llama la atención la pobreza de las oficinas de la planeación urbana intentando determinar y caracterizar el desarrollo urbano ignorando la capacidad de las infraestructuras urbanas para satisfacer las demandas del crecimiento poblacional.

Solamente el desarrollo y mejoramiento tecnológico de las infraestructuras urbanas permitirán hacer frente a las demandas del crecimiento y mejoramiento de la calidad de vida de sus habitantes.

El "derecho a la ciudad" deja de ser una entelequia y se consolida en la medida que las tecnologías urbanas satisfagan el crecimiento constante de las demandas de servicios en la cantidad y calidad que los habitantes demandan.

El diseño de la ciudad, su planificación, su ordenamiento, no pueden seguir siendo un conjunto de definiciones y reglamentaciones formales al margen de los procesos urbanos de la producción de los bienes y servicios que la comunidad demanda y que solo la modernización de las tecnologías urbanas garantiza.

Los Sectores de la producción social, vale decir, primario, secundario y terciario, demandan y desarrollan un complejo conjunto de Tecnologías que permitan satisfacer las demandas del conjunto de la sociedad.

Las arquitecturas territoriales y urbanas demandan de esos sectores, bienes y servicios específicos, a saber: 


\section{El sector primario o extractivo}

Las materias primas, maderas, tierra, metálicas y no metálicas, gravas, piedras, algunas de las cuales participan en el sector de la construcción en forma directa y la mayoría de ellas constituyen insumos básicos para su trasformación en diversidad de productos del sector secundario.

\section{El sector secundario o transformador}

Las cerámicas, los metales en diversidad de presentaciones, las pinturas, los químicos en general, las rocas en la diversidad de presentaciones, los ladrillos, las maderas en sus diferentes gamas y los plásticos en su inmensidad de presentaciones. Todos estos productos necesarios a la construcción.

En este sector transformador aparecen todos los productos de las ingenierías y arquitecturas, con una diversidad de procesos y productos que reclaman tecnologías adecuadas a la diversidad de la oferta y la demanda.

Las empresas constructoras hacen parte de este sector, independiente de su capital y de su recurso humano.

Las tecnologías utilizadas por estas empresas constructoras garantizan la eficiencia y rentabilidad de sus procesos productivos y darán cuenta de la calidad de sus productos.

\section{El sector terciario o de servicios}

Aportará en primer lugar capital y apoyo financiero a la construcción en general y permitirá la aparición de tecnología de alta eficiencia en la medida en que apalanca la calidad de equipos de alta eficiencia y la implantación de procesos de punta y economías de escala.

Las consultorías de ingeniería, urbanismo y arquitectura igualmente de este sector, permiten que sus empresas especializadas de alta eficiencia orienten los procesos y productos del sector secundario.

Lamentablemente solo los ingenieros han desarrollado empresas de consultoría que se ocupan a profundidad de la preingeniería, de la prefactibilidad incluida la simulación de procesos financieros y tecnológicos hasta la definición final, puesto en obra y evaluación.

Los arquitectos han estado ausentes de la consultoría. Sin participación en los estudios de prefactibilidad, en el diseño tecnológico, en la simulación financiera y escasamente ofreciendo consultoría en la prefiguración y en el diseño formal.

Seguramente, un cambio en la organización empresarial de los arquitectos, brindando asesoría en la totalidad de servicios para una correcta inversión social, incluidos los estudios de prefactibilidad, el diseño del proceso tecnológico, el diseño formal estructural, la simulación de la puesta en obra y socialización del producto, darían un vuelco en la calidad de los productos urbano arquitectónicos.

Este cambio en el desarrollo social de la actividad profesional de los arquitectos deberá, necesariamente, empezar en las escuelas de arquitectura, entrenando a los futuros profesionales en la formulación compleja del proceso de producción urbano y arquitectónico. Complejidad ésta que no se compadece con la pobreza actual del ejercicio profesional de nuestros colegas. 
¿Cuáles serían los cambios significativos que bien valdría la pena asumir en los procesos de enseñanza en las escuelas de arquitectura?

En primer lugar, resulta necesario introducir el concepto de que la arquitectura y el urbanismo son inversiones de interés social y que por tanto implican una alta responsabilidad frente a la comunidad. Esa responsabilidad se expresaría en una alta precisión y claridad en los documentos propios de un proyecto de tal carácter y que por tanto esos documentos que lo describen no pueden prestarse a interpretación o a dudas.

¿Cuáles son los documentos mínimos de un proyecto de interés social para la enseñanza de arquitectura?

1. La prefactibilidad:

La prefactibilidad es el documento básico (anteproyecto) que simula el total de las decisiones en condiciones de primera aproximación. El estudiante de arquitectura debe aprender esta práctica y las escuelas de arquitectura deben desarrollar esta experticia; la primera aproximación formal para incursionar en los procesos tecnológicos propios de una inversión social.

2. La composición formal y el proceso tecnológico:

La composición formal debe expresar simultáneamente el proceso de "hacer", de construir de la forma, incluyendo la definición de las prácticas constructivas y los ritmos de ejecución que han de materializar la forma.

3. La estabilidad de la propuesta:

Paralelamente con la composición formal, con la definición de la trama compositiva, el estudiante debe expresar la conducta estructural, el comportamiento de estabilidad de las partes y el todo.

4. El proceso tecnológico y de inversión:

El estudiante debe aprender a expresar los ritmos de construcción y de inversión.

La obra no se ejecuta en un día y debe ser el resultado de un proceso preestablecido.

El proceso tecnológico debe ser la armonización entre ejecución e inversión y por tanto el documento tecnológico debe presentar los flujos de ejecución e inversión. Los ritmos de ejecución expresarán el plan compositivo.

5. Especificaciones y cantidad:

Del proceso tecnológico se deduce la necesidad de expresar la cantidad y calidad de las partes en un documento que contenga la definición de todos los componentes del proyecto de forma tal que no se preste a dudas o interpretaciones.

Si la escuela de arquitectura introduce unas exigencias rigurosas en la elaboración del proyecto de estudio, podemos estar seguros, de que en la vida profesional podrá enfrentar "Proyectos Sociales" con el rigor necesario, sino que estará en condiciones de ofrecer en empresas de consultoría al sector público y privado una arquitectura cada vez más acorde con las calidades que la sociedad tiene derecho a esperar. 